\title{
A Tripodal Aminothioether Ligand Scaffold: Synthesis and Coordination to Zirconium and Hafnium
}

Velabo Mdluli, ${ }^{a}$ Patrick J. Hubbard, ${ }^{a}$ Andrew Kuznicki, ${ }^{a}$ James A. Golen, ${ }^{a}$ Arnold L. Rheingold, ${ }^{b}$ David R. Manke*a

\footnotetext{
${ }^{a}$ Department of Chemistry and Biochemistry, University of Massachusetts Dartmouth, 285 Old Westport Road, North Dartmouth, MA 02747, USA

${ }^{b}$ Department of Chemistry, University of California, San Diego, 9500 Gilman Drive, La Jolla, CA 92093, USA
}

\section{*Corresponding Author:}

David R. Manke

dmanke@umassd.edu

Ph: (508)910-6554

Fax: (508)999-9167

285 Old Westport Rd, Violette 201B

North Dartmouth, MA 02747 


\begin{abstract}
The heptadentate ligand, tris-(2-(2-(methylthio)phenylamino)ethyl)amine (2), has been synthesized from the condensation of nitrilotriacetyl chloride with 2-(methylthio)aniline, to generate 2,2',2"'-nitrilotris( $N$-(2-(methylthio)phenyl)acetamide) (1), followed by a lithium aluminum hydride reduction. The zirconium (3) and hafnium (4) complexes of this ligand were generated via transamination reactions. Both complexes are isostructural, exhibiting a hexadentate $\mathrm{N}_{4} \mathrm{~S}_{2}$ coordination from the ligand, with one diethylamido ligand also bound. The solid state structures of all compounds are reported.
\end{abstract}

\title{
1. Introduction
}

The secondary coordination sphere can have substantial impact on the reactivity observed at a metal center. This local environment can influence binding, kinetics, redox potentials, proton and electron transfer without a direct metal interaction [1-3]. The tris(2-aminoethyl)amine (tren) ligand has been prevalent in transition metal chemistry over the past 25 years [4-6], and the tripodal amine motif has been used as the primary metal binding site in many small molecule secondary coordination sphere studies [7-12]. Our lab has an interest in exploring sulfur donor sites in the secondary coordination sphere and has begun exploring tren based ligands with pendant thioether groups.

Sulfur containing tren ligands are not unknown, with a few $\mathrm{N}_{4} \mathrm{~S}_{3}$ ligands reported in the literature; these systems have shown modest success in the binding of ions, and as neutral amine donors for late transition metals [13-16]. In contrast to the work previously performed, we sought to deprotonate our ligand and use it as a tris(amido)amine, stabilizing higher oxidation state transition metals. Our initial targets were complexes of group 4 metals, particularly zirconium and hafnium. The chemistry of tren based ligands with zirconium is well established [17], and 
such complexes have demonstrated interesting reactivity in recent years, particularly in the area of main group-carbon bond formation [18-20].

\section{Results and Discussion}

\subsection{Ligand Syntheses}

Tris-(2-(2-(methylthio)phenylamino)ethyl)amine was synthesized via standard coupling and reduction techniques using nitrilotriacetic acid (NTA) as the starting material (Scheme 1). The NTA is first converted to nitrilotriacetyl chloride with phosphorus pentachloride [21], which is then coupled to 2-(methylthio)aniline to yield 2,2',2'-nitrilotris $(N-(2-$ (methylthio)phenyl)acetamide (1). The tris-amide is then reduced with lithium aluminum hydride to generate the desired tren-based $\mathrm{N}_{4} \mathrm{~S}_{3}$ ligand (2). The ${ }^{1} \mathrm{H}$ and ${ }^{13} \mathrm{C}$ NMR for both complexes 1 and 2 possess a single set of resonances indicating three equivalent arms for each compound in solution. The coupling step of this synthesis is modular and can produce a variety of tris(aryl)tren

ligands by simply changing the aniline used, on which we will expand in future publications. The synthesis has the advantage of beginning with NTA, while most tris(aryl)tren ligands are currently synthesized via Buchwald couplings to the more expensive tris(2-aminoethyl)amine [22].

\subsection{Ligand Structures}

The solid state structure of (1) is shown in Figure 1. The three arms of the molecule are extended, and one intramolecular $(\mathrm{N} 1-\mathrm{H} 1 \cdots \mathrm{N} 4)$ and two intermolecular $(\mathrm{N} 2-\mathrm{H} 2 \cdots \mathrm{O} 3$; N3$\mathrm{H} 3 \cdots \mathrm{O} 2$ ) hydrogen bonds are observed. The intermolecular hydrogen bonds connect the molecules in an infinite chain along the $a$-axis. The structure is consistent with that observed for the parent nitrilotriacetanilide [23]. 
By contrast, the solid state structure of the reduced ligand (2) exhibits a folded arrangement, similar to that expected upon tripodal coordination of a metal center (Figure 2). This arrangement has been observed for the structures of other aryl substituted tren ligands [24-26]. The compound crystallizes in two different polymorphs: one with a rhombohedral cell (2a) and one with an orthorhombic cell (2b). Both structures possess three intramolecular $\mathrm{N}-\mathrm{H} \cdots \mathrm{S}$ hydrogen bonds $(\mathrm{d}(\mathrm{N} \cdots \mathrm{S})=2.8-3.0 \AA)$ that hold the structures in the folded arrangement. Polymorph 2a has crystallographically imposed three-fold symmetry, with one third of the ligand present in the asymmetric unit. In polymorph $\mathbf{2 b}$, one of the methyl groups is turned down, breaking the three-fold symmetry, but is otherwise isostructural.

\subsection{Metalation Chemistry}

In our first attempts to put a metal center into the binding pocket of this new ligand, we pursued conventional transamination chemistry $[17,27,28]$. Tetrakis(diethylamido)zirconium (IV) and tetrakis(diethylamido)hafnium (IV) were mixed with (2) in toluene and refluxed. After removing solvent and washing, the compounds were isolated in good yield as white solids. The molecules

were characterized by ${ }^{1} \mathrm{H}$ and ${ }^{13} \mathrm{C} \mathrm{NMR}$, indicating a symmetric coordination to the metal center with three equivalent ligand arms, which varies from the solid state structure. There is broadening observed in the ${ }^{1} \mathrm{H}$ spectra which suggests fluxional behavior that would be consistent with the structure observed in the crystal (vide infra). The resonances for a single bound diethylamido ligand is also observed.

\subsection{Zirconium and Hafnium Structures}

Single crystals of $\mathbf{3}$ and $\mathbf{4}$ were grown from a tetrahydrofuran/pentane solution. The molecular structure of $\mathbf{3}$ demonstrates a hexadentate $\mathrm{N}_{4} \mathrm{~S}_{2}$ coordination of the ligand and one diethylamido group still bound to yield seven coordinate zirconium $\left(\mathrm{N}_{5} \mathrm{~S}_{2}\right)$. The nitrogen coordination exhibits 
bond distances and angles similar to the only other reported tris(aryl)tren zirconium amido complex [28], with a slight elongation of the $\mathrm{Zr}-\mathrm{N}$ amido bonds $(\mathrm{d}(\mathrm{Zr}-\mathrm{N})=2.108-2.124 \AA$ vs. $\mathrm{d}(\mathrm{Zr}-\mathrm{N})=2.128-2.198 \AA$ ). The two $\mathrm{Zr}-\mathrm{S}$ bonds show distances of 2.834 and $2.857 \AA$, which are significantly longer than the $\mathrm{Zr}-\mathrm{S}$ distances observed in other similar amido-thioether complexes (2.727-2.786 ̊) [29,30], indicating a weak interaction. This is consistent with the NMR data which possesses only one set of methyl, aryl and methylene resonances in the ${ }^{1} \mathrm{H}$ and ${ }^{13} \mathrm{C}$ spectra, suggesting that these interactions are fluxional. The structure of $\mathbf{4}$ is nearly identical, demonstrating a unit cell, bond distances and angles that are all very similar to $\mathbf{3}$ (Figure 3 ).

\section{Conclusions}

A new $\mathrm{N}_{4} \mathrm{~S}_{3}$ aminothioether ligand has been synthesized and structurally characterized. The ligand is produced via standard condensation and reduction techniques. Transamination chemistry leads to the formation of zirconium and hafnium complexes, which have also been crystallographically characterized. These complexes demonstrate a coordination number of seven with the ligand exhibiting hexadentate $\left(\mathrm{N}_{4} \mathrm{~S}_{2}\right)$ coordination and a single diethylamido ligand also bound. The two sulfur atoms bound to the metal center have relatively long $\mathrm{M}-\mathrm{S}$ distances, indicating a weak interaction. That one thioether site is unbound and that the NMR data indicates that the bonding of these functionalities are fluxional suggests that they should be available for reactivity in the secondary coordination sphere. The synthesis of this ligand is modular and amenable to the production of a variety of substituted tris(aryl)tren ligands. In future publications, we will explore the derivatives of this ligand and its coordination chemistry with other transition metals.

\section{Experimental}

\subsection{General Considerations}


All organic manipulations were carried out using standard Schlenk techniques and metalations were performed in a nitrogen glovebox unless otherwise noted. Pentane, tetrahydrofuran, and toluene were all dried over sodium/benzophenone and distilled prior to use. Chloroform- $d$ was dried over calcium sulfate, distilled and stored over $3 \AA$ molecular sieves. All other solvents and chemicals were used as purchased without further purification. NMR spectra were recorded on a Bruker AVANCE III HD $400 \mathrm{MHz}$ instrument. ${ }^{1} \mathrm{H}$ and ${ }^{13} \mathrm{C}$ NMR chemical shifts were referenced to residual solvent. 2,2',2"'-nitrilotriacetylchloride was prepared according to literature procedure [21]. Elemental analyses were carried out at Robertson Microlit Laboratories in Ledgewood, NJ.

\subsection{X-ray crystallography}

All operations were performed on a Bruker D8 Venture CMOS diffractometer or a Bruker Apex2 CCD diffractometer. Data collection was carried out using the Bruker APEX2 software. Cell refinement and data reduction were performed with the SAINT program [31]. The structure solution was done with SHELXS97 [32] and structure refinement was performed with SHELXL2014 [33]. Further refinement and molecular graphics were generated using the OLEX2 software [34]. Crystallographic data and refinement parameters are included in Table 1 with more detailed information available in the supplementary material.

\subsection{Synthesis of 2,2',2'-Nitrilotris(N-(2-(methylthio)phenyl)acetamide,} $\mathrm{N}\left(\mathrm{CH}_{3} \mathrm{CONC}_{6} \mathrm{H}_{4} \mathrm{SCH}_{3}\right)_{3}(\mathbf{1})$.

To a cooled $\left(0^{\circ} \mathrm{C}\right) 100 \mathrm{~mL}$ THF solution of 2,2',2”-nitrilotriacetyl chloride (3.30 g, $\left.13.7 \mathrm{mmol}\right)$ was added a mixture of 2-methylthioaniline $(6.0 \mathrm{~mL}, 44.0 \mathrm{mmol})$ and trimethylamine $(5.33 \mathrm{~mL}$, $42.5 \mathrm{mmol}$ ) dropwise over the course of 15 minutes. The resulting mixture was allowed to warm to room temperature, stirred for 12 hours, filtered and solvent was removed in vacuo. The 
resulting oil was dissolved in dichloromethane $(100 \mathrm{~mL})$ and extracted with water $(3 \times 100 \mathrm{~mL})$. The organic layer was dried with anhydrous sodium sulfate, filtered and solvent was removed in vасио. The crude product was triturated with a minimal amount of ethanol, filtered and washed with pentane to afford a white solid $(6.19 \mathrm{~g}, 81 \%) .{ }^{1} \mathrm{H} \mathrm{NMR}\left(400 \mathrm{MHz}, \mathrm{CDCl}_{3}\right): \delta 9.30(\mathrm{~s}, 3 \mathrm{H}$, $\mathrm{NH}), 8.18(\mathrm{~d}, J=7.6 \mathrm{~Hz}, 3 \mathrm{H}, \mathrm{ArH}), 7.40(\mathrm{~d}, J=7.6 \mathrm{~Hz}, 3 \mathrm{H}, \mathrm{ArH}), 7.26(\mathrm{~m}, 3 \mathrm{H}, \operatorname{Ar} H), 7.11(\mathrm{t}, J$ $=7.3 \mathrm{~Hz}, 3 \mathrm{H}, \mathrm{ArH}), 3.75\left(\mathrm{~s}, 6 \mathrm{H}, \mathrm{CH}_{2}\right), 2.25\left(\mathrm{~s}, 9 \mathrm{H}, \mathrm{CH}_{3}\right),{ }^{13} \mathrm{C} \mathrm{NMR}\left(100 \mathrm{MHz}, \mathrm{CDCl}_{3}\right): \delta 167.6$ (OC), $137.0(\mathrm{ArC}), 132.6(\mathrm{ArC}), 128.2(\mathrm{ArC}), 127.5(\mathrm{ArC}), 125.3(\mathrm{ArC}), 121.9(\mathrm{ArC}), 59.9$ $\left(C \mathrm{H}_{2}\right), 18.5\left(\mathrm{CH}_{3}\right)$. Elemental analysis calcd. for $\mathrm{C}_{27} \mathrm{H}_{30} \mathrm{~N}_{4} \mathrm{O}_{3} \mathrm{~S}_{3}:$ C 58.46, H 5.45, N 10.1; Found: C 58.30, H 5.24, N 10.05

4.4. Synthesis of tris-(2-(2-(methylthio)phenylamino)ethyl)amine, $\mathrm{N}\left(\mathrm{CH}_{3} \mathrm{CH}_{2} \mathrm{NC}_{6} \mathrm{H}_{4} \mathrm{SCH}_{3}\right)_{3}$ (2).

To a cooled $\left(0^{\circ} \mathrm{C}\right) 100 \mathrm{~mL}$ solution of $\mathbf{1}(3.37 \mathrm{~g}, 6.07 \mathrm{mmol})$ was added $\mathrm{LiAlH}_{4}(2.51 \mathrm{~g}, 66.8$ mmol) in ten parts. The reaction mixture was heated at reflux for 12 hours. The mixture was cooled to $0{ }^{\circ} \mathrm{C}$, quenched with $20 \mathrm{~mL}$ of water, then treated with a $\mathrm{NaOH}$ solution $(20 \% \mathrm{w} / \mathrm{w}, 30$ $\mathrm{mL})$, and stirred for $1 \mathrm{~h}$. The mixture was filtered, the precipitate washed with THF (50 mL) and the filtrate was stripped of solvent under vacuum. The resulting solid residue was dissolved in dichloromethane $(100 \mathrm{~mL})$, then extracted with water $(3 \times 100 \mathrm{~mL})$. The organic layer was dried with anhydrous sodium sulfate, and solvent was removed in vacuo. The resulting crude product was triturated with a minimal amount of ethanol, filtered, and washed with pentane to afford a white-yellow solid product (1.18 g, 38\%). ${ }^{1} \mathrm{H} \mathrm{NMR}\left(400 \mathrm{MHz}, \mathrm{CDCl}_{3}\right): \delta 7.32(\mathrm{~d}, J=7.6 \mathrm{~Hz}$, 3H, ArH), $7.11(\mathrm{t}, J=7.7 \mathrm{~Hz}, 3 \mathrm{H}, \operatorname{ArH}), 6.64(\mathrm{t}, J=7.4,3 \mathrm{H}, \operatorname{ArH}), 6.56(\mathrm{~d}, J=7.9 \mathrm{~Hz}, 3 \mathrm{H}$, $\operatorname{ArH}), 5.27(\mathrm{t}, J=4.6 \mathrm{~Hz}, 3 \mathrm{H}, \mathrm{NH}), 3.29\left(\mathrm{q}, J=5.9 \mathrm{~Hz}, 6 \mathrm{H}, \mathrm{CH}_{2}\right), 2.89\left(\mathrm{t}, J=6.1 \mathrm{~Hz}, 6 \mathrm{H}, \mathrm{CH}_{2}\right)$, $2.18\left(\mathrm{~s}, 9 \mathrm{H}, \mathrm{CH}_{3}\right) ;{ }^{13} \mathrm{C} \mathrm{NMR}\left(100 \mathrm{MHz}, \mathrm{CDCl}_{3}\right): \delta 148.0(\mathrm{ArC}), 133.3(\mathrm{ArC}), 129.1(\mathrm{ArC})$, 
$120.7(\operatorname{ArC}), 117.1(\mathrm{ArC}), 110.1(\mathrm{ArC}), 54.4\left(\mathrm{CH}_{2}\right), 42.2\left(\mathrm{CH}_{2}\right), 17.8\left(\mathrm{CH}_{3}\right)$. Elemental analysis calcd. for $\mathrm{C}_{27} \mathrm{H}_{36} \mathrm{~N}_{4} \mathrm{~S}_{3}$ : C 63.24, H 7.08, N 10.93; Found: C 63.41, H 7.13, N 11.10

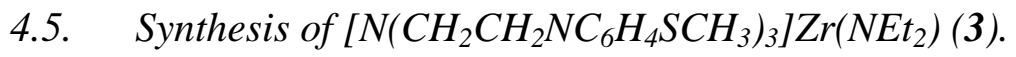

To tris-(2-(2-(methylthio)phenylamino)ethyl)amine (214mg, $417 \mu \mathrm{mol})$ in toluene $(4 \mathrm{~mL})$ was added tetrakis(diethylamido)zirconium $(150 \mu \mathrm{L}, 486 \mu \mathrm{mol})$. The resulting reaction mixture was refluxed overnight, dried under vacuum and washed with pentane to give a white solid (142 mg, 75\%). ${ }^{1} \mathrm{H}$ NMR (400 MHz, $\left.\mathrm{CDCl}_{3}\right): \delta 7.17(\mathrm{~d}, J=7.2 \mathrm{~Hz}, 3 \mathrm{H}, \mathrm{Ar} H), 7.06(\mathrm{t}, J=7.2 \mathrm{~Hz}, 3 \mathrm{H}$, $\operatorname{Ar} H$ ), 6.62 (m, 6H, $\mathrm{ArH}$ ), 3.69 (br s, 6H, $\mathrm{CH}_{2}$ ), 3.33 (br s, 4H, $\mathrm{CH}_{2}$ ), 2.84 (br s, $6 \mathrm{H}, \mathrm{CH}_{2}$ ), 2.18 (s, 9H, SCH$), 0.17\left(\mathrm{t}, J=7.9 \mathrm{~Hz}, 6 \mathrm{H}, \mathrm{CH}_{3}\right) ;{ }^{13} \mathrm{C} \mathrm{NMR}\left(100 \mathrm{MHz}, \mathrm{CDCl}_{3}\right): \delta 158.9(\mathrm{ArC}), 130.7$ ( $\operatorname{ArC}), 128.5(\operatorname{ArC}), 126.2(\mathrm{ArC}), 117.9(\mathrm{ArC}), 117.7(\mathrm{ArC}), 55.9\left(\mathrm{CH}_{2}\right), 51.0\left(\mathrm{CH}_{2}\right), 42.7\left(\mathrm{CH}_{2}\right)$, $20.3\left(\mathrm{SCH}_{3}\right), 12.8\left(\mathrm{CH}_{3}\right)$. Elemental analysis calcd. for $\mathrm{C}_{31} \mathrm{H}_{43} \mathrm{ZrN}_{5} \mathrm{~S}_{3}$ : C 55.32, H 6.44, N 10.40; Found: C 55.05, H 6.14, N 10.16.

\subsection{Synthesis of $\left[\mathrm{N}\left(\mathrm{CH}_{2} \mathrm{CH}_{2} \mathrm{NC}_{6} \mathrm{H}_{4} \mathrm{SCH}_{3}\right)_{3}\right] \mathrm{Hf}\left(\mathrm{NEt} \mathrm{t}_{2}\right)(4)$.}

To tris-(2-(2-(methylthio)phenylamino)ethyl)amine (156 mg, $304 \mu \mathrm{mol})$ in toluene $(3 \mathrm{~mL})$ was added tetrakis(diethylamido)hafnium $(100 \mu \mathrm{L}, 322 \mu \mathrm{mol})$. The resulting reaction mixture was refluxed overnight, dried under vacuum and washed with pentane to give a white solid (164 mg, 70\%). ${ }^{1} \mathrm{H}$ NMR (400 MHz, $\left.\mathrm{CDCl}_{3}\right): \delta 7.20(\mathrm{~d}, J=7.6 \mathrm{~Hz}, 3 \mathrm{H}, \mathrm{ArH}), 7.08(\mathrm{t}, J=7.7 \mathrm{~Hz}, 3 \mathrm{H}$, $\mathrm{ArH}$ ), 6.64 (m, 6H, $\mathrm{ArH}$ ), 3.75 (br s, 6H, $\mathrm{CH}_{2}$ ), 3.36 (br s, 4H, $\mathrm{CH}_{2}$ ), 2.80 (br s, 6H, $\mathrm{CH}_{2}$ ), 2.20 (s, 9H, $\left.\mathrm{SCH}_{3}\right), 0.13\left(\mathrm{t}, J=7.9 \mathrm{~Hz}, 6 \mathrm{H}, \mathrm{CH}_{3}\right) ;{ }^{13} \mathrm{C} \mathrm{NMR}\left(100 \mathrm{MHz}, \mathrm{CDCl}_{3}\right): \delta 159.1(\mathrm{ArC}), 130.9$ ( $\operatorname{ArC}), 128.6(\operatorname{ArC}), 126.5(\mathrm{ArC}), 118.7(\mathrm{ArC}), 117.9(\mathrm{ArC}), 54.9\left(\mathrm{CH}_{2}\right), 50.6\left(\mathrm{CH}_{2}\right), 42.5\left(\mathrm{CH}_{2}\right)$, $20.7\left(\mathrm{SCH}_{3}\right), 12.8\left(\mathrm{CH}_{3}\right)$. Elemental analysis calcd. for $\mathrm{C}_{31} \mathrm{H}_{43} \mathrm{HfN}_{5} \mathrm{~S}_{3}: \mathrm{C} 48.97$, H 5.70, N 9.21; Found: C 49.20, H 5.47, N 9.11.

\section{Appendix A. Supplementary data}


CCDC 15502327-15502331 contain the supplementary crystallographic data for $\mathbf{1}, \mathbf{2 a}, \mathbf{2 b}, \mathbf{3}$ and 4. These data can be obtained free of charge via http://www.ccdc.cam.ac.uk/conts/retreiving/html, or from the Cambridge Crystallographic Data Centre, 12 Union Road, Cambridge CB2 1EZ, UK; fax: (+44) 1223-336-033; or e-mail: deposit@ccdc.cam.ac.uk.

\section{Acknowledgements}

We gratefully acknowledge funding for this work from the National Science Foundation (CHE1229339 and CHE-1429086). PJH would like to thank the UMass Dartmouth Office of Undergraduate Research for support. AK is grateful for support from the Urban Massachusetts Louis Stokes Alliance for Minority Participation. 
Table 1. Crystallographic data collection and refinement parameters for 1, 2a, 2 b, 3 and 4.

\begin{tabular}{|c|c|c|c|c|c|}
\hline Compound & 1 & $2 \mathbf{a}$ & $2 \mathbf{b}$ & 3 & 4 \\
\hline CCDC \# & 1502328 & 1502327 & 1502330 & 1502329 & 1502331 \\
\hline Empirical formula & $\mathrm{C}_{27} \mathrm{H}_{30} \mathrm{~N}_{4} \mathrm{O}_{3} \mathrm{~S}_{3}$ & $\mathrm{C}_{27} \mathrm{H}_{36} \mathrm{~N}_{4} \mathrm{~S}_{3}$ & $\mathrm{C}_{27} \mathrm{H}_{36} \mathrm{~N}_{4} \mathrm{~S}_{3}$ & $\mathrm{C}_{31} \mathrm{H}_{43} \mathrm{~N}_{5} \mathrm{~S}_{3} \mathrm{Zr}$ & $\mathrm{C}_{31} \mathrm{H}_{43} \mathrm{HfN}_{5} \mathrm{~S}_{3}$ \\
\hline Formula weight & 554.73 & 512.78 & 512.78 & 673.1 & 760.37 \\
\hline $\mathrm{T}(\mathrm{K})$ & 200 & 100 & 100 & 120 & 120 \\
\hline$\lambda(\AA)$ & 1.54178 & 0.71073 & 0.71073 & 0.71073 & 0.71073 \\
\hline Crystal system & Triclinic & trigonal & orthorhombic & triclinic & triclinic \\
\hline Space group & $P 1$ & P31c & $P 2_{1} 2_{1} 2_{1}$ & $P 1$ & $P 1$ \\
\hline \multicolumn{6}{|l|}{ Unit cell parameters } \\
\hline$a(\AA)$ & $8.5161(3)$ & $12.6005(8)$ & $10.4020(13)$ & $8.9019(19)$ & $8.889(2)$ \\
\hline$b(\AA)$ & $12.5469(4)$ & $12.6005(8)$ & $12.5438(14)$ & $11.051(3)$ & $11.031(3)$ \\
\hline$c(\AA)$ & $14.4605(4)$ & $9.6577(6)$ & $20.097(2)$ & $16.839(4)$ & $16.845(4)$ \\
\hline$\alpha\left(^{\circ}\right)$ & $66.547(1)$ & 90 & 90 & $74.507(9)$ & 74.351(9) \\
\hline$\beta\left(^{\circ}\right)$ & $85.114(2)$ & 90 & 90 & $84.912(9)$ & $84.815(9)$ \\
\hline$\gamma\left({ }^{\circ}\right)$ & $73.979(2)$ & 120 & 90 & $75.785(9)$ & $75.724(8)$ \\
\hline $\mathrm{V}\left(\AA^{3}\right)$ & $1361.88(8)$ & $1327.94(19)$ & $2622.2(5)$ & $1547.0(6)$ & $1540.9(7)$ \\
\hline $\mathrm{Z}$ & 2 & 2 & 4 & 2 & 2 \\
\hline$D_{\text {calc }}\left(\mathrm{g} / \mathrm{cm}^{3}\right)$ & 1.353 & 1.282 & 1.299 & 1.445 & 1.639 \\
\hline Absorption coefficient $\left(\mathrm{mm}^{-1}\right)$ & 2.785 & 0.302 & 0.306 & 0.588 & 3.618 \\
\hline $\mathrm{F}(000)$ & 584 & 548 & 1096 & 704 & 768 \\
\hline Crystal dimensions (mm) & $0.20 \times 0.05 \times 0.05$ & $0.35 \times 0.24 \times 0.18$ & $0.10 \times 0.08 \times 0.05$ & $0.22 \times 0.18 \times 0.10$ & $0.18 \times 0.15 \times 0.10$ \\
\hline Crystal color & colorless & colorless & colorless & yellow & colorless \\
\hline No. of measured/independent reflections & $17138 / 5008$ & $6170 / 1802$ & $22533 / 6241$ & $56617 / 5893$ & $34273 / 5845$ \\
\hline No. of reflections with $\mathrm{I}>2 \sigma(\mathrm{I})$ & 3897 & 1711 & 5502 & 5307 & 5539 \\
\hline No. of parameters & 349 & 107 & 319 & 366 & 366 \\
\hline Final $R_{1}[\mathrm{I}>2 \sigma(\mathrm{I})]$ & 0.0462 & 0.0272 & 0.0357 & 0.0225 & 0.0179 \\
\hline Final $w R_{2}\left(\right.$ on $\mathrm{F}^{2}$, all data $)$ & 0.1217 & 0.0612 & 0.0802 & 0.0579 & 0.0425 \\
\hline Goodness-of-fit (GOF) on $\mathrm{F}^{2}$ & 1.031 & 1.047 & 1.028 & 1.073 & 1.066 \\
\hline Largest diff. peak and hole $\left(\mathrm{e} \AA^{-3}\right)$ & $0.34 /-0.28$ & $0.18 /-0.14$ & $0.27 /-0.23$ & $0.33 /-0.30$ & $0.42 /-0.55$ \\
\hline
\end{tabular}




\section{References}

[1] S.A. Cook, A.S. Borovik, Acc. Chem. Res. 48 (2015) 2407.

[2] S.A. Cook, E.A. Hill, A.S. Borovik, Biochemistry 54 (2015) 4167.

[3] A.S. Borovik, Acc. Chem. Res. 38 (2005) 54.

[4] C.C. Cummins, R.R. Schrock, W.M. Davis, Organometallics 11 (1992) 1452.

[5] R.R. Schrock, Acc. Chem. Res. 30 (1997) 9.

[6] R.R. Schrock, Acc. Chem. Res. 38 (2005) 955.

[7] C.E. MacBeth, A.P. Golobek, V.G. Young Jr., C. Yang, K. Kuczera, M.P. Hendrich, A.S. Borovik, Science 289 (2000) 938.

[8] R.L. Lucas, M.K. Zart, J. Murkerjee, T.N. Sorrell, D.R. Powell, A.S. Borovik, J. Am. Chem. Soc. 128 (2006) 15476.

[9] Y.J. Park, J.W. Ziller, A.S. Borovik, J. Am. Chem. Soc. 133 (2011) 9258.

[10] S.J. Tereniak, R.K. Carlson, L.J. Clouston, V.G. Young Jr., E. Bill, R. Maurice, Y.-S. Chen, H.J. Kim, L. Gagliardi, C.C. Lu, J. Am Chem. Soc. 136 (2014) 1842.

[11] E.M. Matson, Y.J. Park, A.R. Fout, J. Am. Chem. Soc. 136 (2014) 17398.

[12] C.M. Wallen, J. Bacsa, C.C. Scarborough, J. Am. Chem. Soc. 137 (2015) 14606.

[13] G. Steel, A. Mustapha, J. Reglinski, A.R. Kennedy, Polyhedron 67 (2014) 360.

[14] P.J. Brothers, C.E.F. Rickard, C.M. Strange, D.C. Ware, Aust. J. Chem. P.J. Brothers, C.E.F. Rickard, C.M. Strange, D.C. Ware, Aust. J. Chem. 50 (1997) 373.

[15] M. Işiklan, M.A. Saeed, A. Pramanik, B.M. Wong, F.R. Fronczek, Md.A. Hossain, Cryst. Growth Des. 11 (2011) 959.

[16] A.-S. Jullien, C. Gateau, C. Lebrun, I. Kieffer, D. Testemale, P. Delangle, Inorg. Chem. 53 (2014) 5229.

[17] C. Morton, I.J. Munslow, C.J. Sanders, N.W. Alcock, P. Scott, Organometallics 18 (1999) 4608.

[18] S.N. MacMillan, J.M. Tanski, R. Waterman, Chem. Commun. (2007) 4172.

[19] A.F. Maddox, J.J. Davidson, T. Shalumova, J.M. Tanski, R. Waterman, Inorg. Chem. 52 (2013) 7811.

[20] A.J. Roering, S.E. Leshinski, S.M. Chan, T. Shalumova, S.N. MacMillan, J.M. Tanski, R. Waterman, Organometallics 29 (2010)2557.

[21] T.M. Klaptöke, B. Krumm, R.Z. Moll, Naturforsch. B. 68 (2013) 735.

[22] G.E. Greco, R.R. Schrock, Inorg. Chem. 40 (2001) 3850.

[23] J.M. Rowland, G.J. Ibanez, M.M. Olmstead, M. Ruf, P.K. Mascharak, Acta Cryst. E57, o1001.

[24] A. Almesäker, J.L. Scott, L. Spiccia, C.R. Strauss, Tetrahedron Letters 50 (2009) 1847. 
[25] Y.S. Moroz, M.K. Takase, P. Müller, E.V. Rybak-Akimova, Acta Cryst. E67 (2011) o3421.

[26] A. Wünsche von Leupoldt, C. Förster, T.J. Fiedler, N.H. Bings, K. Heinze, Eur. J. Inorg. Chem. (2013) 6079.

[27] Z. Duan, A.A. Naiini, J.-H. Lee, J.G. Verkade, Inorg. Chem. 34 (1995) 5477.

[28] C. Morton, K.M. Gillespie, C.J. Sanders, P. Scott, J. Organomet. Chem. 606 (2000) 141.

[29] X. Wang, Z. Chen, X.-L. Sun, Y. Tong, Z. Xie, Org. Lett. 13 (2011) 4758.

[30] D.D. Graf, R.R. Schrock, W.M. Davis, R. Stumpf, Organometallics 18 (1999) 843.

[31] Bruker (2014) APEX2, SAINT and SADABS. Bruker AXS Inc., Madison, Wisconsin, USA.

[32] Sheldrick, G.M. Acta Cryst. A64 (2008) 112.

[33] Sheldrick, G.M. Acta Cryst. C71 (2015) 3.

[34] Dolomanov, O.V, Bourhis, L.J., Gildea, R.J., Howard, J.A.K., Puschmann, H. J. Appl. Cryst. 42 (2009) 339. 

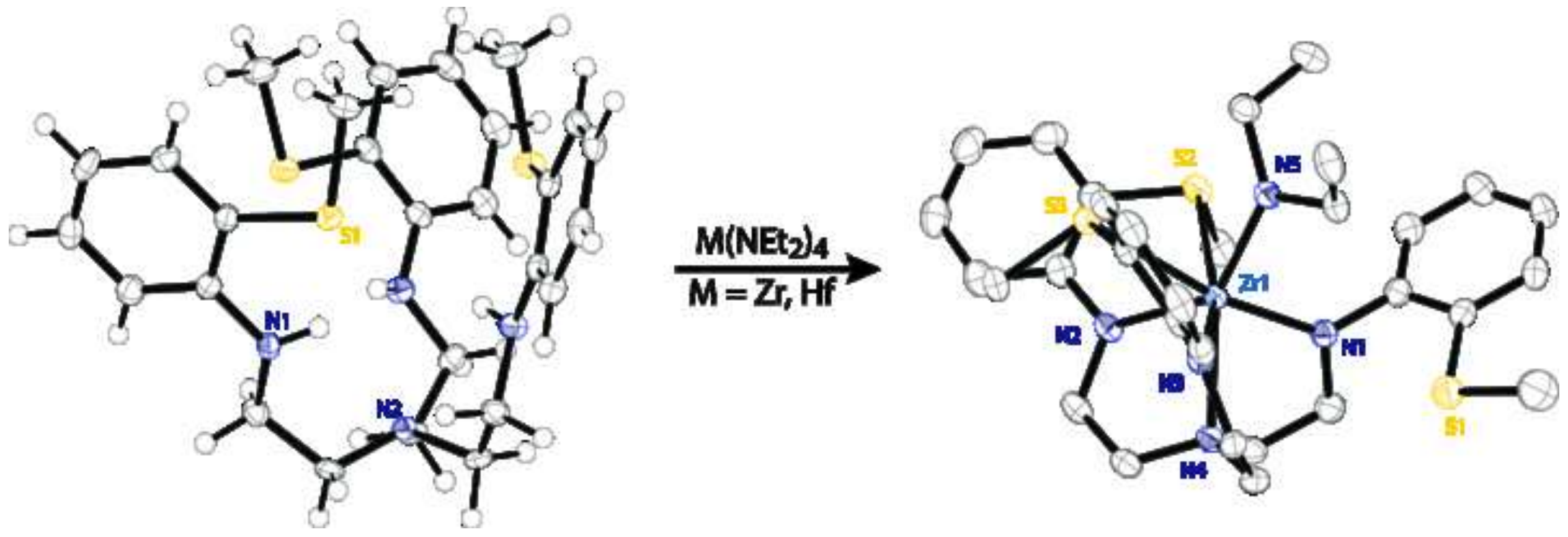